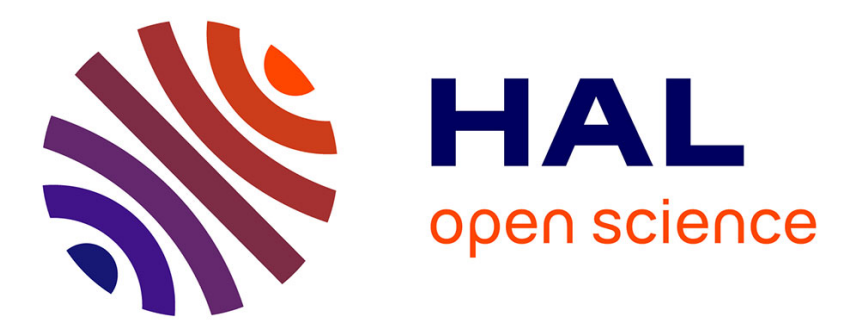

\title{
Magnetic field frustration of the metal-insulator transition in $\mathrm{V} 2 \mathrm{O} 3$
}

J. Trastoy, J del Valle, Y Kalcheim, D. Ravelosona, M. J Rozenberg, Ivan K Schuller, A Camjayi, J.-P Crocombette, D. A Gilbert, J A Borchers, et al.

\section{- To cite this version:}

J. Trastoy, J del Valle, Y Kalcheim, D. Ravelosona, M. J Rozenberg, et al.. Magnetic field frustration of the metal-insulator transition in V 2 O 3. Physical Review B, 2020, 10.1103/PhysRevB.101.245109 . hal-03024677

\section{HAL Id: hal-03024677 \\ https://hal.science/hal-03024677}

Submitted on 25 Nov 2020

HAL is a multi-disciplinary open access archive for the deposit and dissemination of scientific research documents, whether they are published or not. The documents may come from teaching and research institutions in France or abroad, or from public or private research centers.
L'archive ouverte pluridisciplinaire HAL, est destinée au dépôt et à la diffusion de documents scientifiques de niveau recherche, publiés ou non, émanant des établissements d'enseignement et de recherche français ou étrangers, des laboratoires publics ou privés. 


\title{
Magnetic field frustration of the metal-insulator transition in $\mathrm{V}_{2} \mathrm{O}_{3}$
}

\author{
J. Trastoy $\odot,{ }^{1,2, *}$ A. Camjayi, ${ }^{3}$ J. del Valle,,${ }^{1,}$ Y. Kalcheim, ${ }^{1}$ J.-P. Crocombette, ${ }^{4}$ D. A. Gilbert $\odot,{ }^{5,6}$ J. A. Borchers, ${ }^{5}$ \\ J. E. Villegas $\odot,{ }^{2}$ D. Ravelosona, ${ }^{7}$ M. J. Rozenberg,,${ }^{1,8}$ and Ivan K. Schuller ${ }^{1}$ \\ ${ }^{1}$ Department of Physics and Center for Advance Nanoscience, University of California San Diego, La Jolla, California 92093, USA \\ ${ }^{2}$ Unité Mixte de Physique, CNRS, Thales, Université Paris-Sud, Université Paris Saclay, 91767 Palaiseau, France \\ ${ }^{3}$ Departamento de Física, FCEyN, UBA and IFIBA, Conicet, Pabellón 1, Ciudad Universitaria, 1428 CABA, Argentina \\ ${ }^{4}$ CEA, DEN, Service de Recherches de Métallurgie Physique, Université Paris-Saclay, F-91191 Gif-sur-Yvette, France \\ ${ }^{5}$ NIST Center for Neutron Research, Gaithersburg, Maryland 20899, USA \\ ${ }^{6}$ Department of Materials Science and Engineering, University of Tennessee, Knoxville, Tennessee 37919, USA \\ ${ }^{7}$ Center for Nanoscience and Nanotechnology (C2N), UMR 9001, 91120 Palaiseau, France \\ and Spin-Ion Technologies, 10 Boulevard Thomas Gobert, 91120 Palaiseau, France \\ ${ }^{8}$ Université Paris-Saclay, CNRS, Laboratoire de Physique des Solides, 91405, Orsay, France
}

(Received 16 January 2020; revised manuscript received 8 April 2020; accepted 23 April 2020; published 1 June 2020)

\begin{abstract}
Despite decades of efforts, the origin of metal-insulator transitions (MITs) in strongly correlated materials remains one of the main long-standing problems in condensed-matter physics. An archetypal example is $\mathrm{V}_{2} \mathrm{O}_{3}$, which undergoes simultaneous electronic, structural, and magnetic phase transitions. This remarkable feature highlights the many degrees of freedom at play in this material. In this work, acting solely on the magnetic degree of freedom, we reveal an anomalous feature in the electronic transport of $\mathrm{V}_{2} \mathrm{O}_{3}$ : On cooling, the magnetoresistance changes from positive to negative values well above the MIT temperature, and shows divergent behavior at the transition. The effects are attributed to the magnetic field quenching antiferromagnetic fluctuations above the Néel temperature $T_{N}$, and preventing long-range antiferromagnetic ordering below $T_{N}$. In both cases, suppressing the antiferromagnetic ordering prevents the opening of the incipient electronic gap. This interpretation is supported by Hubbard model calculations which fully reproduce the experimental behavior. Our study sheds light on this classic problem providing a clear and physical interpretation of the nature of the metal-insulator transition.
\end{abstract}

DOI: 10.1103/PhysRevB.101.245109

\section{INTRODUCTION}

Electronic correlations are responsible for a plethora of remarkable phenomena in condensed-matter physics [1-7]. Among the most studied phenomena is the metal-insulator transition (MIT) [2,8-11], where a material that should be metallic at all temperatures according to conventional band theory becomes insulating in a first-order transition with a resistance change of several orders of magnitude. Understanding the MIT has been one of the outstanding challenges in condensed-matter physics in the last decades. A number of theories have been advanced to explain these observations, among which three stand out. Mott proposed [2] that Coulomb repulsion between electrons could split the conduction band by opening a charge gap. Slater [12] argued that the gap could be opened due to magnetic ordering where electronic energies become spin dependent. Peierls [13] suggested that structural changes, such as dimerization, alter the periodicity of the lattice giving rise to a gap.

\footnotetext{
*jtrastoy@physics.ucsd.edu

†Current address: Department of Quantum Matter Physics, University of Geneva, Quai Ernest-Ansermet 24, 1205 Geneva, Switzerland.
}

Disentangling these three main mechanisms is a complicated task. No other material reflects this as well as $\mathrm{V}_{2} \mathrm{O}_{3}$ $[11,14,15]$, which has become a classic strongly correlated physics problem. Three transitions occur concurrently involving the three different aspects suggested by Mott, Slater, and Peierls: an electronic, a magnetic, and a structural transition, respectively. More specifically, cooling below $160 \mathrm{~K}, \mathrm{~V}_{2} \mathrm{O}_{3}$ undergoes a transition from a paramagnetic metal with rhombohedral structure to an antiferromagnetic (AF) insulator with a monoclinic unit cell. Neutron diffraction [16] showed that AF fluctuations above the transition are observed at a wave vector different from the one present in the low- $T$ ordered state, which was interpreted as evidence for orbital order. After almost 50 years from the discovery of the MIT transition, numerous aspects remain a matter of debate.

In the present study we report a strong coupling between magnetism and electronic transport in $\mathrm{V}_{2} \mathrm{O}_{3}$ across the MIT. The magnetoresistance (MR) shows an anomalous behavior upon cooling from high temperature to the transition temperature, $T_{\mathrm{MIT}}$. The MR is small and positive at room temperature; it decreases upon cooling, changes sign well above $T_{\mathrm{MIT}}$, and shows a negative enhancement at the transition. A Hubbard model calculation captures the qualitative behavior indicating that the anomalous MR results from a magnetic frustration effect, which prevents the opening of the electronic gap. Our 


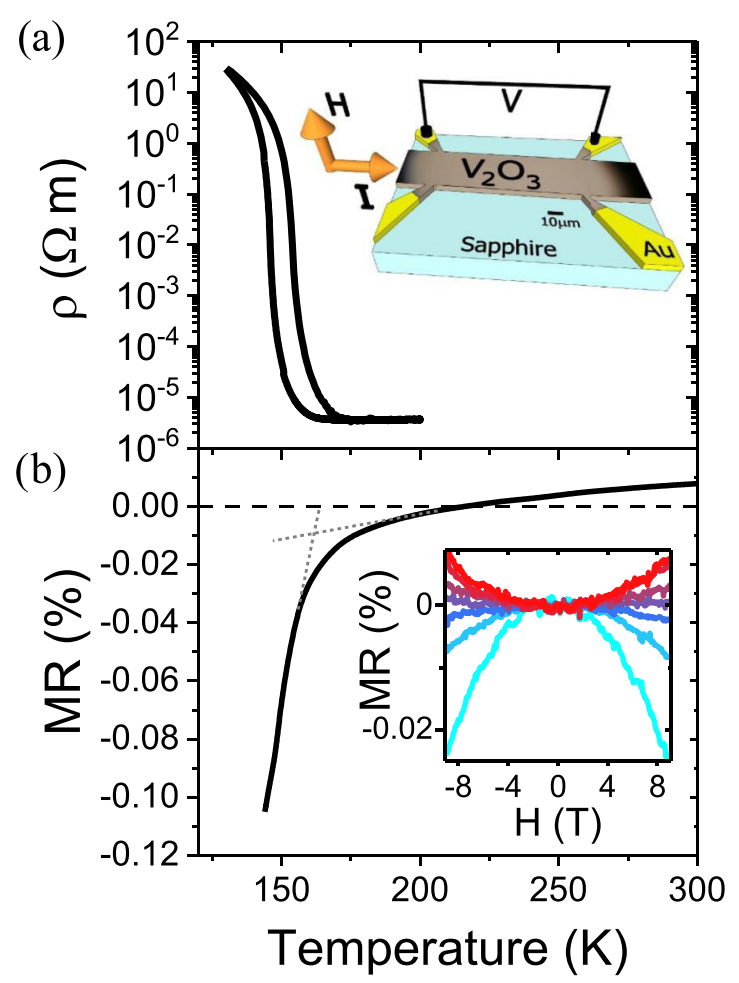

FIG. 1. The metal-insulator transition (MIT) and its associated magnetoresistance (MR). Resistivity $\rho$ (a) and MR at $9 \mathrm{~T}$ (b) as a function of temperature for a virgin $\mathrm{V}_{2} \mathrm{O}_{3}$ microbridge. The MR curve shows that a crossover from positive to negative values above the MIT takes place, and seems to diverge as the transition takes place. The gray dotted lines are a guide to the eyes indicating the two MR behaviors before and after the transition. Insets: (a) Optical microscope image of the $\mathrm{V}_{2} \mathrm{O}_{3}$ microbridge inserted in a sketch showing the measurement configuration. Four-lead magnetoresistance measurements were performed in a magnetic field applied out of plane and perpendicular to the current. The voltage is measured between the longitudinal contacts. (b) The quadratic dependence of MR vs field $H$ at several temperatures from $280 \mathrm{~K}$ (red) to $160 \mathrm{~K}$ (light blue) for the same $\mathrm{V}_{2} \mathrm{O}_{3}$ sample.

work provides key physical insight on the long-standing question of the nature of the MIT in $\mathrm{V}_{2} \mathrm{O}_{3}$.

\section{EXPERIMENTAL RESULTS}

$\mathrm{V}_{2} \mathrm{O}_{3}$ thin films $100 \mathrm{~nm}$ thick were grown by rf magnetron sputtering deposition epitaxially along the (012) direction on $r$-cut sapphire substrates. A detailed account of the sample deposition and characterization can be found elsewhere [17]. Eight films grown at the same time were used for this experiment. Two were left in the virgin state while the others were blanket irradiated with increasing doses of $100-\mathrm{keV} \mathrm{He}^{+}$ions: $2 \times 10^{14}, 8 \times 10^{14}, 2 \times 10^{15}$, and $8 \times 10^{15} \mathrm{ions} / \mathrm{cm}^{2}$. Five films were patterned with $40-\mu \mathrm{m}$-wide and $100-\mu \mathrm{m}$-long microbridges (shown in Fig. 1) for in-plane electrical transport, via photolithography and reactive ion etching $\left(\mathrm{Ar}+\mathrm{Cl}_{2}\right)$. $\mathrm{Ti} / \mathrm{Au}$ electrodes were deposited using $e$-beam evaporation and lift-off. Comparisons between the pristine film and the microbridge showed that etching did not impact the material properties. All electrical transport measurements were per- formed in a Quantum Design Dynacool PPMS ${ }^{1}$ equipped with a 9-T magnet. A Keithley $6221^{1}$ current source was used in conjunction with a Keithley $2182 \mathrm{~A}^{1}$ nanovoltmeter behind two Keithley $6517 \mathrm{~A}^{1}$ electrometers acting as a buffer. This allows measuring high resistances without having the relatively low internal resistance of the nanovoltmeter in parallel with the sample, and minimizes the measurement noise.

The temperature-dependent resistivity, $\rho$, for a $\mathrm{V}_{2} \mathrm{O}_{3}$ bridge is shown by the black curve in Fig. 1(a). The MIT temperature is defined as the temperature at which the resistance achieves a positive $d \rho / d T$. The MIT occurs around $150 \mathrm{~K}$ accompanied by a resistivity change of six orders of magnitude. This resistivity change is comparable with the one found in bulk single crystals [18], which confirms the high quality of our thin films.

In order to explore the magnetic degree of freedom during the MIT, we measured the magnetoresistance (MR) of different $\mathrm{V}_{2} \mathrm{O}_{3}$ microbridges in which the MIT temperature was controllably varied. The main panel of Fig. 1(b) shows the temperature dependence of the MR, as measured relative to $9 \mathrm{~T}: \mathrm{MR}=[\rho(9 \mathrm{~T})-\rho(0)] / \rho(0)$. This field is far smaller than the exchange interaction [19]. The inset of Fig. 1(b) shows a series of resistivity vs magnetic field, $H$, curves at different temperatures, with $H$ applied perpendicular to the plane. Qualitatively similar results were obtained for in-plane field measurements parallel and perpendicular to the current $\vec{I}$ (not shown). Note that here it is not possible to scan the temperature at different fixed fields to determine the MR due to its relatively small magnitude. As expected for ordinary metals [20], at high temperatures the MR is small and positive with a quadratic field dependence. Upon cooling from $300 \mathrm{~K}$, the MR decreases and changes signs, becoming negative at $T \approx 200 \mathrm{~K}$, well above the MIT. As the temperature is decreased further, the MR becomes more negative and shows a steep enhancement at $T_{\mathrm{MIT}}$. Below the MIT, the sample becomes highly insulating and the MR can no longer be measured due to a poor signal-to-noise ratio.

To further investigate the anomalous behavior of the MR, we used $\mathrm{He}^{+}$ion irradiation to locally disorder the $\mathrm{V}_{2} \mathrm{O}_{3}$, which has been previously shown as an effective technique to tune $T_{\mathrm{MIT}}$ [15]. The precise evaluation of the structural change due to irradiation is beyond the scope of this work. Several simultaneously grown $\mathrm{V}_{2} \mathrm{O}_{3}$ films were irradiated with increasing dose, $\delta$, of $100-\mathrm{keV} \mathrm{He}^{+}$ions: $2 \times 10^{14}, 8 \times 10^{14}$, $2 \times 10^{15}$, and $8 \times 10^{15}$ ions $\mathrm{cm}^{-2}$. Figure 2(a) confirms the expected trend: Increasing the fluence shifts the MIT to lower temperatures by $4.7 \mathrm{~K} /\left(10^{14}\right.$ ions $\left.\mathrm{cm}^{-2}\right)$ without affecting the magnitude of the resistance change up to the highest dose (orange curve). The highest-dose sample shows no apparent MIT, but does possess a small hysteretic feature in the resistivity [see inset of Fig. 2(a)], likely indicating the presence of domains which still undergo a MIT. MR measurements of

${ }^{1}$ Certain commercial equipment, instruments, or materials are identified in this manuscript in order to specify the experimental procedure adequately. Such identification is not intended to imply recommendation or endorsement by the National Institute of Standards and Technology, nor is it intended to imply that the materials or equipment are necessarily the best available for the purpose. 

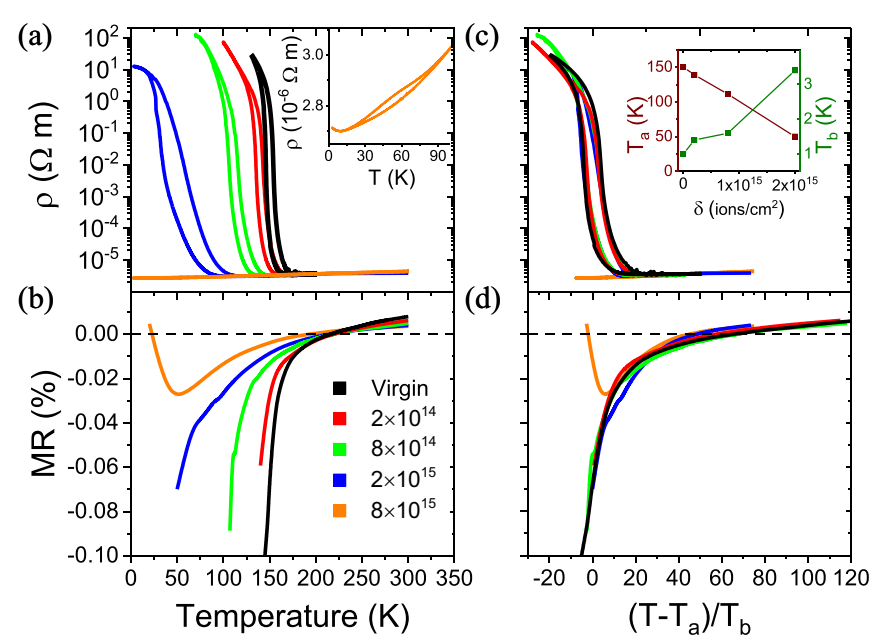

FIG. 2. The metal-insulator transition (MIT) and its associated magnetoresistance (MR). Resistivity $\rho$ (a) and MR at $9 \mathrm{~T}$ (b) as a function of temperature for a series of $\mathrm{V}_{2} \mathrm{O}_{3}$ samples irradiated with increasing $\mathrm{He}^{+}$doses (in units of ions $\mathrm{cm}^{-2}$ ). The $\rho$ and MR curves are displaced to lower temperatures for increasing irradiation dose. (c), (d) show the collapse obtained for $\rho$ and MR, respectively, when $T$ is scaled to $\left(T-T_{a}\right) / T_{b}$. Insets: (a) Detail of the $\rho(T)$ curve for the highest-irradiation sample, which shows the hysteretic behavior, a hallmark of the first-order MIT. (c) $T_{a}$ and $T_{b}$ scaling parameters as a function of the irradiation dose. The evolution is linear.

the irradiated samples, Fig. 2(b), show a similar enhancement at $T_{\mathrm{MIT}}$ and also a shift to lower temperatures, commensurate with the shift in the MIT. For the highest-dose sample [orange curve in Fig. 2(b)], the absence of a full MIT allows us to measure the MR even below the MIT (see Supplemental Material [21]). The steep enhancement of the MR upon cooling is still present and, surprisingly, it is followed by a decrease in the MR toward 0 . Interestingly, the change in the sign of the MR occurs at $\approx 200 \mathrm{~K}$ for all of the samples, seemingly insensitive to the irradiation disorder. Also the MIT and MR data from any of the samples can be scaled and translated $\left[T \rightarrow\left(T-T_{a}\right) / T_{b}\right]$ to overlap onto a single master curve, shown in Figs. 2(c) and 2(d), respectively. Perform- ing this scaling, the parameters $T_{a}$ and $T_{b}$ follow a simple linear dependence on $\delta$, as shown in the inset of Fig. 2(c). This indicates that the transition temperature $T_{\mathrm{MIT}}$ decreases linearly with $\delta$.

Neutron diffraction measurements were performed on single $0.5 \mathrm{~cm}^{2} \times 100$-nm-thick thin-films of pristine and irradiated $\mathrm{V}_{2} \mathrm{O}_{3}$ using the BT-4 and BT-7 triple axis spectrometers at the NIST Center for Neutron Research with $14.7-\mathrm{meV}$ $(2.35-\AA)$ neutrons. The sample was cooled in a closed-cycle refrigerator with a silicon-diode temperature sensor located near the sample location. The (010) and (020) peaks were simultaneously captured with in situ measurements of the resistance, while heating the samples from low temperature. The (010) peak appears only as a result of the magnetic (AF) ordering, as shown, for example, in Fig. 3(a), while the (020) nuclear peak shifts through the structural transition. Thus these two measurements, combined with the in situ resistance, capture the magnetic, structural, and MIT transitions. The peak intensity of the (010) can be used to fit an order parameter, as shown, for example, in Fig. 3(b), and determine $T_{N}$. Resistance measurements from the neutron experiment agree well with the MIT results in Fig. 1, allowing the neutron and MR results to be compared. For the pristine sample, $T_{N}$ and the structural transition temperature, $T_{S}$, are commensurate with the MIT. With increased irradiation, $T_{N}=T_{S}$ shifts to lower temperatures along with the onset of the MIT. Note that the $T_{\mathrm{MIT}}$ in irradiated samples, as determined during the neutrons measurements, occurs at a temperature slightly lower than $T_{N}$, although within the approximate error of the measurement, and is likely due to percolation effects. In Fig. 3(c) we present the results for $T_{N}$ as a function of the irradiation dose. The data show a reduction of $T_{N}$ and $T_{S}$ that is also linear in $\delta$, in agreement with the MIT results.

\section{DISCUSSION}

Our results show a unique MR profile in $\mathrm{V}_{2} \mathrm{O}_{3}$, with a positive MR far above $T_{N}$, which crosses over to negative values below $200 \mathrm{~K}$ and exhibits a steep enhancement below $T_{\mathrm{MIT}}$ and $T_{N} . \mathrm{He}^{+}$irradiation shifts $T_{\mathrm{MIT}}, T_{N}$, and $T_{S}$ (a)

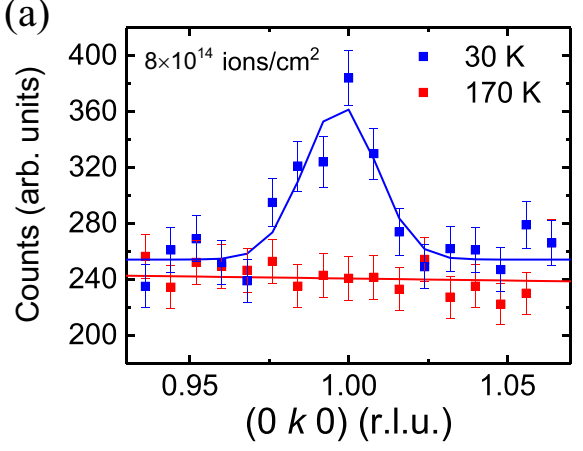

(b)

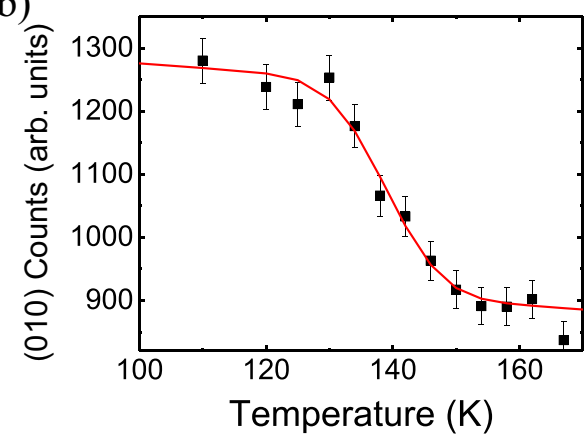

(c)

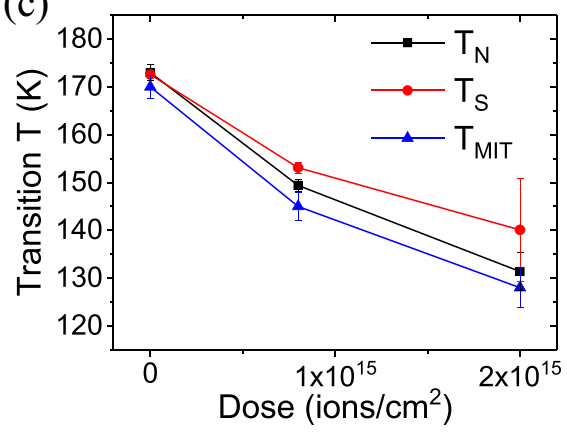

FIG. 3. Neutron diffraction measurements. (a) Neutron diffraction along the $(0 k 0)$ direction for the sample irradiated with $8 \times$ $10^{14}$ ions $/ \mathrm{cm}^{2}$. The blue data, measured at low temperature, show a peak at $(010)$ corresponding to the antiferromagnetic ordering of $\mathrm{V}_{2} \mathrm{O}_{3}$. Nothing is observed at high temperature. The blue curve is a Gaussian fit to the data. (b) (010) diffraction peak intensity as a function of temperature for the same sample. The red line is a guide to the eye. Error bars are representative of the uncertainty for the intensity data. (c) Linear decrease of the Néel $\left(T_{N},\right)$, MIT $\left(T_{\mathrm{MIT}}\right)$, and structural transition $\left(T_{S}\right)$ temperatures with increasing irradiation dose. Errors in transition temperatures estimated from least-square fits. 
linearly with $\delta$ (see Supplemental Material [21]), along with the steep negative enhancement in MR. However, it does not significantly affect the crossover of the MR at $\approx 200 \mathrm{~K}$. The facts that the resistivity and MR curves can be collapsed via a simple rescaling [Figs. 2(c) and 2(d)], and that neutrons show a reduction hand-in-hand of $T_{\mathrm{MIT}}, T_{N}$, and $T_{S}$ [Fig. 3(c)], establish that irradiation systematically modifies the critical temperatures but does not change the underlying physics. The discussion below presents the MR behavior above $T_{N}$ as a result of quenching local AF fluctuations, while the MR enhancement at $T_{N}$ is attributed to field-driven suppression of the AF transition, which is reproduced by mean-field Hubbard modeling.

Far above $T_{\mathrm{MIT}}$ and $T_{N}$, the MR is positive, as is common for metals [20]. The negative MR below $\approx 200 \mathrm{~K}$ is consistent with the presence of additional scattering which can be suppressed by a magnetic field. The magnitude of the MR increases as the temperature is decreased [Fig. 2(b)] implying that the number, size, or strength of the scattering sources is increasing approaching $T_{N}$ and the MIT. Since the sign change of the MR occurs above $T_{N}$ where no long-range magnetic order is present, the scattering can instead be attributed to locally ordered AF fluctuations, which have been previously reported in this material using neutron scattering by Bao et al. [16]. Note that similar measurements are not feasible for these thin films due to the limited sample volume. Interestingly, the MR crossover is robustly insensitive to irradiation disorder, in contrast to $T_{N}$. This behavior is consistent with Bao's work, which showed that $\mathrm{AF}$ fluctuations in $\mathrm{V}_{2} \mathrm{O}_{3}$ are independent of vanadium deficiency and are decoupled from $T_{N}$. Thus, from all these considerations it seems natural to assume that the $\mathrm{AF}$ fluctuations are the source of the electronic scattering and that they get quenched by the application of an external magnetic field, accounting for the negative MR.

While magnetic fluctuations can qualitatively account for the MR behavior above the transition, the AF correlation length diverges below $T_{N}$. To try to rationalize the experimental observations, we performed a study of the MR at the onset of the AF MIT within a Hubbard model. Dynamical mean-field theory (DMFT) calculations were done using a continuous-time quantum Monte Carlo impurity solver [22], based on the hybridization expansion and with a semicircular noninteracting density of states. A one-band antiferromagnetic self-consistency with applied external magnetic field was implemented. A moderate value for the electronic interactions $U / W=0.85$ was adopted, where $U$ is the Coulomb repulsion and $W$ the bandwidth (with $W=4 t$ and $t$ the hopping amplitude). To avoid the inherent problems of analytical continuation of Monte Carlo data, the conductivity was obtained from the Kubo formula in bosonic Matsubara frequencies [23-25], and its zero-frequency value was extrapolated. To obtain the density of states, a maximal entropy numerical method was used. Special care was taken to obtain well converged solutions in the vicinity of the magnetic transition. Although it does not incorporate the complexities of the actual lattice structure, the Hubbard model has provided a basic description of the paramagnetic MIT in Cr-doped $\mathrm{V}_{2} \mathrm{O}_{3}$ [24] and of the critical behavior at the end point of the first-order transition line [26]. Hall-effect measurements at room temperature indicate there is one carrier per vanadium atom in the metallic state of the virgin and irradiated samples (see Supplemental Material [21], Fig. S1). Thus, we consider a Hubbard model at half filling. This model captures the paramagnetic metal-antiferromagnetic insulator transition as a function of temperature. Note that local density approximation (LDA)+DMFT calculations for $\mathrm{V}_{2} \mathrm{O}_{3}$ are impractical for the present study due to the significant technical issues involved in a calculation across the MIT with long-range ordering and magnetic field. The calculation in the Hubbard model is still a challenge due to the phenomenon of critical slowing down across the MIT, requiring very long CPU time to achieve the numerical convergence and precision required to obtain the conductivity and the MR.

Figure 4 shows the results of the model calculations. The systematic exploration (not shown) of the ratio $U / W$, where $U$ is the Coulomb interaction and $W$ the bandwidth, shows that the best agreement is found for $U / W \sim 1$. This places the model in the correlated regime, consistent with previous work $[26,27,24]$. In our calculations we adopt an external magnetic field in the direction of the AF magnetization ( $z$ direction). While this choice is made for numerical simplicity, we note that in the experiments there is a component of the field along the AF ordering direction. Figure 4(a) shows that the model reproduces several of the main qualitative experimental features of the MR. At high $T$, in the paramagnetic metallic state the MR is small and positive, and at the MIT undergoes a steep enhancement toward negative values. As noted above, the model cannot capture the crossover in the magnetization above $T_{N}$, since it originates from fluctuations that are not included in the mean-field model.

In our calculations of MR from the Hubbard model, we have used a large value of the magnetic field $H=0.05 \mathrm{~W} / \mu_{B}$, which corresponds to $\sim 180 \mathrm{~T}$ and is required to achieve good numerical precision. Nevertheless, we can extract the magnitude of the MR at the experimental magnetic field of $9 \mathrm{~T}$ since, in agreement with the experiments [inset of Fig. 1(b)], the calculated $\operatorname{MR}(H)$ behaves as $H^{2}$ [inset of Fig. 4(a)]. Thus, we obtain $\operatorname{MR}(9 \mathrm{~T})=(9 \mathrm{~T} / H)^{2} \times$ $\operatorname{MR}(H)=\left[9 \mathrm{~T} /\left(0.05 W / \mu_{B}\right)\right]^{2} \times 80 \% \approx 0.2 \%$, in remarkably good order-of-magnitude agreement.

The DMFT simulations provide physical insight pointing to the mechanism that can explain the experimentally observed enhancement of the MR near $T_{N}$. The origin of this feature can be traced to the frustration of the magnetic moments in one of the two Néel sublattices. In Fig. 4(b) we plot the staggered antiferromagnetic moment $m=-\left\langle S_{z}\right\rangle \mu_{B} g_{S} / \hbar$ at $H=0$ (black line) and $H=0.05 \mathrm{~W} / \mu_{B}$ (red and blue lines for the A and B sublattice, respectively), where $\left\langle S_{z}\right\rangle$ is the mean value of the $z$ component of the spin polarization of one sublattice and $g_{s}=-2$ is the gyromagnetic factor. As the system is cooled through $T_{N}$ in the absence of an external magnetic field, the two sublattices order antiferromagnetically; i.e., $\left|m_{A}\right|=\left|m_{B}\right|=m_{A F}$ [see sketch at the bottom of Fig. 4(c)]. However, applying an external field, say, in the $+z$ direction, favors the upward polarization $m_{A}$, while at the $B$ sites, which order antiparallel to the $A$ sites, the applied field $H$ competes with the microscopic Weiss field due to superexchange interactions with the nearest-neighbor $A$ sites. This is revealed by the simulations as seen in Fig. 4(b), where $m_{A}$ (red line) develops its full staggered moment, while $m_{B}$ 

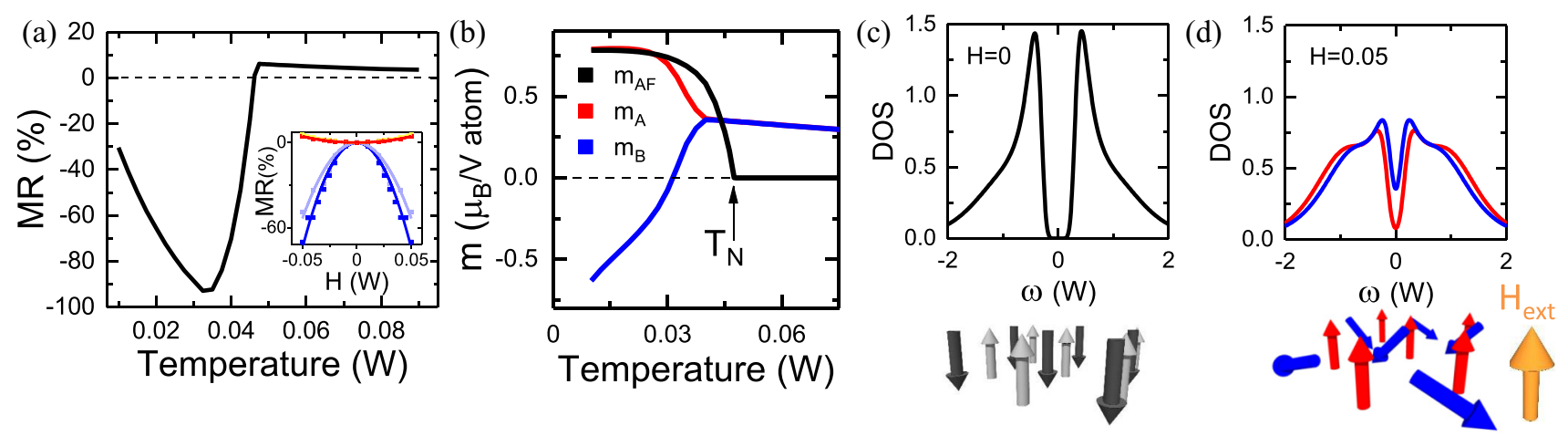

FIG. 4. The calculated effect of the magnetic field on the antiferromagnetic (AF) ordering. (a) Calculated magnetoresistance as a function of temperature using DMFT calculations of the Hubbard model. Inset: Calculated magnetoresistance (squares) for $T(W)=0.07$ (red), 0.05 (yellow), 0.0425 (light blue), and 0.04 (blue). Lines are parabolic fits, showing the dependence with field is quadratic as in the experiments. (b) Calculated evolution as a function of temperature of the AF staggered moment $m_{\mathrm{AF}}$ (black line) in zero field, and the two magnetic sublattices $m_{A}$ (red line) and $m_{B}$ (blue line) under a magnetic field $H=0.05$ parallel to sublattice A. All moments are in units of the Bohr magneton $\mu_{B}$ per $\mathrm{V}$ atom. Under a magnetic field, sublattice B does not develop its full moment due to the frustration from the magnetic field. The difference in spin ordering is depicted at the bottom of (c),(d). In the absence of an applied field, all spins (in black) order antiferromagnetically. When an external field (orange arrow) is applied along the A sublattice (red spins), the B sublattice (blue spins) is disordered. The calculated density of states (DOS) at $T=0.025$ is shown for the case of zero field (c) and $H=0.05$ (d), revealing how the magnetic field produces a closing of the AF gap, more pronounced for sublattice B (blue line) than for sublattice A (red line).

(blue line) fails to establish its downward polarization. Thus, near the Néel temperature the magnetic moments of sublattice $B$ are frustrated $\left(m_{B} \sim 0\right)$ and fail to polarize [see sketch at the bottom of Fig. 4(d)]. This has implications in the density of states (DOS): For $H=0$ [Fig. 4(c)] the electronic gap is opened, whereas with an applied field [Fig. 4(d)] the gap is filled. Accordingly, the magnetic field prevents the AF ordering and lowers the resistivity, which leads to a large, enhanced, negative MR in the vicinity of $T_{N}$, as found experimentally.

This behavior can also be understood from energy considerations. Near $T_{N}$ the AF moments are small, $m \approx 0$ and the quadratic energy gain of the superexchange interactions, $m^{2} J$, is dominated by the linear energy gain of the external magnetic field, $m H$. However, at lower $T, m$ continues to grow as long-range $\mathrm{AF}$ order develops, and eventually the quadratic superexchange coupling energy overcomes the external magnetic field energy when $m \gg 0$. At this point the effect of $H$ becomes less significant and the MR gradually returns back to zero, as observed in the model and experimentally in the highest-irradiated sample [orange line Fig. 2(b)].

\section{CONCLUSION}

In summary, our experiments shed light on the longstanding problem of the metal-insulator transition in the correlated system $\mathrm{V}_{2} \mathrm{O}_{3}$. We find an anomalous behavior of the MR that is linked to the MIT. As the system is cooled down from room temperature the MR decreases and changes sign, becoming negative well above $T_{\mathrm{MIT}}$ as the result of locally ordered antiferromagnetic fluctuations [16]. Passing through the magnetic transition temperature the negative MR is enhanced. Supported by Hubbard model calculations, the enhanced MR can be attributed to field-induced frustration in one of the magnetic sublattices that inhibits the long-range AF ordering and consequently suppresses the MIT.

Our model calculations qualitatively reproduce the experimental results considering a transition caused by just
Coulomb and superexchange magnetic interactions, thus a pure Mott-Heisenberg MIT. Under this scenario, the structural transition could be hypothesized to be a consequence of the magnetoelectronic phase transition. This could be explained as a result of the change in the atomic binding forces caused by the localization of the electronic wave function at the MIT. Such a change would enhance the lattice electronic compressibility $[28,29]$, leading to the structural phase transition, where the new lattice symmetry results in a variation of the final antiferromagnetic ordering vector. In fact, in neutron diffraction experiments [16] different $\mathrm{AF}$ wave vectors were observed between the AF fluctuations above the MIT and the long-range AF ordering below the MIT. Those observations remain largely unaccounted for, and may find thus a natural resolution. This scenario may be eventually tested by realistic structure DMFT calculations of phonon spectra. However, due to the many orbitals and the presence of magnetic ordering, such an approach currently remains a challenge.

\section{ACKNOWLEDGMENTS}

We thank Igor Mazin and Noam Bernstein for useful discussions, and George Kassabian for extensive technical help. We also thank Yang Zhao and Zhijun Xu for their assistance with measurements on the BT-7 triple axis spectrometer as well as William Ratcliff for his assistance with measurements on the BT-4 triple axis spectrometer at the NIST Center for Neutron Research. The device fabrication, magnetotransport, and neutron measurements were supported by the Office of Basic Energy Science, U.S. Department of Energy, BESDMS, funded by the Department of Energy's Office of Basic Energy Science, DMR under Grant No. DE FG02 87ER45332, and the synthesis and characterization of the oxides by the Vannevar Bush Faculty Fellowship program sponsored by the Basic Research Office of the Assistant Secretary of Defense for Research and Engineering and funded by the Office of Naval Research through Grant No. N00014-15-1- 
2848. M.J.R. acknowledges support by public grants from the French National Research Agency (ANR) project MoMA and the French-US Associated International Laboratory on Nanoelectronics funded by CNRS. J.E.V. acknowledges support from ERC Grant No. 647100. A.C. gratefully acknowledges support from CONICET and UBACyT. J.T. and J.d.V. would like to thank Fundación Ramón Areces for support through a postdoctoral fellowship.
[1] J. Hubbard, Proc. R. Soc. London, Ser. A 276, 238 (1963).

[2] N. F. Mott, Metal-Insulator Transitions (Taylor \& Francis, London, 1990).

[3] E. Dagotto, Rev. Mod. Phys. 66, 763 (1994).

[4] J. M. Tranquada, B. J. Sternlieb, J. D. Axe, Y. Nakamura, and S. Uchida, Nature 375, 561 (1995).

[5] J. C. Loudon, N. D. Mathur, and P. A. Midgley, Nature 420, 797 (2002).

[6] N. S. Bingham, M. H. Phan, H. Srikanth, M. A. Torija, and C. Leighton, J. Appl. Phys. 106, 023909 (2009).

[7] A. Georges, L. de' Medici, and J. Mravlje, Annu. Rev. Condens. Matter Phys. 4, 137 (2013).

[8] J. B. Goodenough, J. Solid State Chem. 3, 490 (1971).

[9] J. M. Honig and L. L. V. Zandt, Annu. Rev. Mater. Sci. 5, 225 (1975).

[10] J. B. Torrance, P. Lacorre, A. I. Nazzal, E. J. Ansaldo, and C. Niedermayer, Phys. Rev. B 45, 8209 (1992).

[11] M. Imada, A. Fujimori, and Y. Tokura, Rev. Mod. Phys. 70, 1039 (1998).

[12] J. C. Slater, Phys. Rev. 82, 538 (1951).

[13] R.E. Peierls, Quantum Theory of Solids (Oxford University Press, 2001).

[14] F. J. Morin, Phys. Rev. Lett. 3, 34 (1959).

[15] J. G. Ramirez, T. Saerbeck, S. Wang, J. Trastoy, M. Malnou, J. Lesueur, J.-P. Crocombette, J. E. Villegas, and I. K. Schuller, Phys. Rev. B 91, 205123 (2015).

[16] W. Bao, C. Broholm, G. Aeppli, P. Dai, J. M. Honig, and P. Metcalf, Phys. Rev. Lett. 78, 507 (1997).

[17] J. Trastoy, Y. Kalcheim, J. del Valle, I. Valmianski, and I. K. Schuller, J. Mater. Sci. 53, 9131 (2018).
[18] D. B. McWhan and J. P. Remeika, Phys. Rev. B 2, 3734 (1970).

[19] J. C. Leiner, H. O. Jeschke, R. Valentí, S. Zhang, A. T. Savici, J. Y. Y. Lin, M. B. Stone, M. D. Lumsden, J. Hong, O. Delaire, W. Bao, and C. L. Broholm, Phys. Rev. X 9, 011035 (2019).

[20] A. B. Pippard, Magnetoresistance in Metals (Cambridge University Press, Cambridge, 1989).

[21] See Supplemental Material at http://link.aps.org/supplemental/ 10.1103/PhysRevB.101.245109 for more information on the irradiation-dose dependence of the transition temperature, the inhomogeneity in the highest-irradiated sample, the carrier concentration, and the calculation of the imaginary part of the self-energy.

[22] E. Gull, A. J. Millis, A. I. Lichtenstein, A. N. Rubtsov, M. Troyer, and P. Werner, Rev. Mod. Phys. 83, 349 (2011).

[23] A. Georges, G. Kotliar, W. Krauth, and M. J. Rozenberg, Rev. Mod. Phys. 68, 13 (1996).

[24] T. Pruschke and R. Zitzler, J. Phys.: Condens. Matter 15, 7867 (2003).

[25] A. Millis, in Strong Interactions In Low Dimensions, edited by D. Baeriswyl and L. Degiorgi (Springer, Dordrecht, 2004), pp. 195-235.

[26] P. Limelette, A. Georges, D. Jérome, P. Wzietek, P. Metcalf, and J. M. Honig, Science 302, 89 (2003).

[27] F. Lechermann, N. Bernstein, I. I. Mazin, and R. Valentí, Phys. Rev. Lett. 121, 106401 (2018).

[28] S. R. Hassan, A. Georges, and H. R. Krishnamurthy, Phys. Rev. Lett. 94, 036402 (2005).

[29] A. Camjayi, R. Chitra, and M. J. Rozenberg, Phys. Rev. B 73, 041103(R) (2006). 